\title{
Line-broadening and anharmonic effects in the attenuated total reflectance infrared spectra of calcite
}

\author{
Etienne BALAN ${ }^{1, *}$, Julie AUFORT $^{1}$, Giuseppe D. SALDI ${ }^{2}$, Christian BROUDER $^{1}$ and Michele LAZZERI ${ }^{1}$ \\ ${ }^{1}$ Sorbonne Université, CNRS, IRD, MNHN, Institut de Minéralogie, de Physique des Matériaux et de \\ Cosmochimie (IMPMC), 4 place Jussieu, 75005 Paris, France \\ *Corresponding author, e-mail: etienne.balan@sorbonne-universite.fr \\ ${ }^{2}$ Géosciences Environnement Toulouse (GET), CNRS/UMR 5563-Université Paul Sabatier, \\ 14 av. E. Belin, 31400 Toulouse, France
}

\begin{abstract}
The attenuated total reflectance (ATR) spectra of two calcite samples have been measured using Ge and diamond internal reflection elements. One of the samples was obtained by precipitation from solution and displays sub-micrometer sized particles. The second sample was obtained by grinding a single-crystal of calcite ("Iceland spar" variety). Theoretical spectra were obtained by computing the reflection coefficient of the interface between the ATR internal reflection element and a homogeneous effective medium representing the powder sample. The dielectric properties of the effective medium have been determined from those of bulk calcite using the Bruggeman approach which is adequate for standard powders with intermediate fractions of solid and empty porosity. Although this modeling approach provides a quantitative interpretation of the broadening of strong absorption bands in the calcite ATR spectra, it fails to reproduce the apparent splitting of the strong $v_{3} \mathrm{CO}_{3}$ band. This problem was solved by combining the electrostatic modeling approach with a semi-quantum model of the dielectric tensor of bulk calcite, which allows for a frequencydependent damping of the $v_{3} \mathrm{CO}_{3}$ excitation. The frequency-dependence is ascribed to a multiphonon-related resonance in the $v_{3}$ $\mathrm{CO}_{3}$ phonon self-energy at a frequency close to its transverse optical frequency. The combination of approaches exposed in the present study makes it possible to discriminate among physically different processes affecting the powder infrared spectra of calcite, some being related to the long-range nature of electrostatic interactions in polar materials and others being related to atomic-scale anharmonic interactions between vibrational modes.
\end{abstract}

Key-words: calcite; infrared spectroscopy; ATR; multi-phonon; effective medium.

\section{Introduction}

Infrared spectroscopy is a versatile method allowing to probe frequency-dependent dielectric properties of minerals and materials in a large variety of geometrical configurations (e.g. Farmer, 1974; McMillan \& Hofmeister, 1988). Focusing on powders, their infrared spectra can be recorded in a transmission configuration after dilution of a small quantity of sample in an infrared transmitting medium, typically potassium bromide. Sample mixing with a powdered infrared transmitting medium is also required to record diffuse reflectance infrared spectra of strongly absorbing samples. In contrast, attenuated total reflectance (ATR) spectroscopy can be applied to pure powders. In this case, absorption-like features are recorded as a modulation of the reflection coefficient of the interface between an infrared reflection element (IRE), made of a highly refractive material, and the packed powder sample. The sample preparation is facilitated, extending the application field of infrared spectroscopy to routine characterization of materials (e.g. Loftus et al., 2015; Lebon et al., 2016). The ATR spectroscopy is also an appropriate technique to investigate the bulk properties of homogeneous isotropic media such as liquids or amorphous compounds (e.g. Dignam \& Mamiche-Afara, 1988; Amma et al., 2015). Classical electromagnetic modeling and Kramers-Kronig transform enable to determine the complex dielectric function of the samples from their ATR spectra (e.g. Dignam \& Mamiche-Afara, 1988; Huang \& Urban, 1992; Boulet-Audet et al., 2010). In the case of powders, additional electrostatic effects occur due to the divided and composite nature of the sample and it becomes challenging to retrieve the intrinsic dielectric properties of crystals from an inversion of powder ATR data, particularly for anisotropic materials.

A direct modeling strategy can be proposed to compute powder ATR spectra of uniaxial materials by combining the effective medium modeling of the powder and the computation of the complex frequency-dependent reflection coefficient of the interface between the isotropic effective medium and the IRE material (Aufort et al., 2016). Application of this approach to synthetic and biologic apatite samples brought quantitative explanations for the peculiar 
shape of strongly absorbing ATR bands and for the differences observed between ATR spectra recorded with a diamond or a Ge IRE (Aufort et al., 2016, 2018).

In the present study, we confront this approach to the modeling of calcite ATR spectra. Calcite $\left(\mathrm{CaCO}_{3}\right.$, space group $R \overline{3} c$ ) is an important industrial material and a key biogenic constituent of the shell of many organisms and animal eggs (e.g. Weiner \& Dove, 2003). It is a well-known uniaxial material used as a benchmark system in infrared spectroscopic studies (e.g. Kristova et al., 2015, Udvardi et al., 2017; Griffiths et al., 2018). Infrared spectroscopy in transmission or ATR geometry is also extensively used to analyze synthetic or natural calcite samples (e.g. Gueta et al., 2007; Poduska et al., 2011; Balan et al., 2017; Jenkins et al., 2018). The present results confirm the effectiveness of the proposed modeling strategy and make it possible to unravel peculiar anharmonic properties affecting the strong carbonate stretching band of the calcite infrared spectrum.

\section{Materials and experimental methods}

Two highly contrasted calcite samples were experimentally investigated. One sample, referred to as "nanocalcite" (Saldi et al., 2018), consists in a sub-micrometer sized synthetic calcite powder precipitated from a supersaturated $\left(\mathrm{NH}_{4}\right)_{2} \mathrm{CO}_{3}+\mathrm{CaCl}_{2}$ solution at $\mathrm{pH} \sim 10$ (Pokrovsky et al., 2004). The powder, as characterized by transmission electron microscopy (TEM, JEOL JEM ARM-200F), is made of well crystallized particles ranging in size from 20 to $250 \mathrm{~nm}$, exhibiting a platy and rounded shape (Saldi et al., 2018). The second sample is a single crystal of natural calcite ("Iceland spar" variety) of unknown origin. Subsets of powder sample were obtained by grinding in ethanol with an agate mortar and pestle for durations ranging from 5 to $120 \mathrm{~min}$. The sample ground for $120 \mathrm{~min}$, referred to as "IS 120", displays particles with an irregular shape and a distribution of size ranging from $\sim 10 \mu \mathrm{m}$ to less than $1 \mu \mathrm{m}$ (Fig. 1). The $120 \mathrm{~min}$ grinding time corresponds to the maximum time above which atomic-scale structural damage becomes discernable in the IR spectra.

The ATR-FTIR spectra were recorded using a Quest ATR device (Specac) and a Nicolet 6700 FTIR spectrometer equipped with an Ever-Glo source, $\mathrm{KBr}$ beamsplitter and DTGS-KBr detector. Pure powder samples were packed at the surface of the ATR IRE and the spectra were recorded between 400 and $4000 \mathrm{~cm}^{-1}$ by averaging 200 scans with a resolution of $1 \mathrm{~cm}^{-1}$. For each sample, two different spectra were recorded using either a diamond or a Ge IRE. The ATR spectra are reported in absorbance units.

\section{Results}

\subsection{Experimental ATR spectra of calcite}

The infrared spectroscopic properties of calcite have been extensively investigated by experiment (e.g. Hellwege et al., 1970; White, 1974; Posch et al., 2007; Udvardi

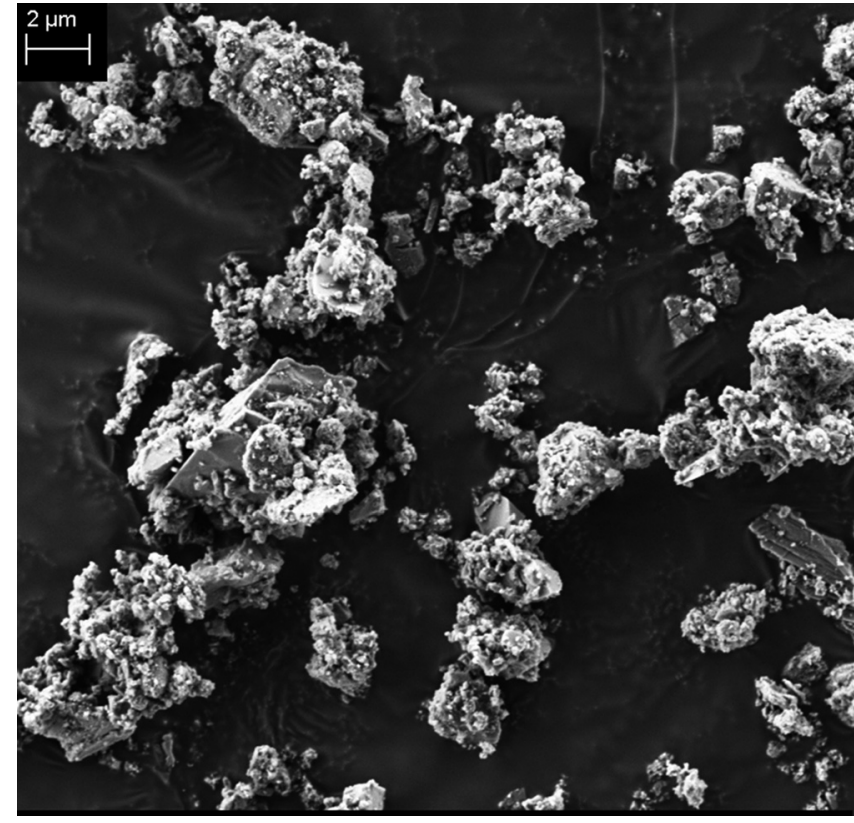

Fig. 1. Scanning electron micrograph (Zeiss Ultra 55 SEM, secondary electron mode) of the calcite single crystal ground for 120 min (IS 120 sample). Note the irregular shape of particles and the broad range of particle sizes.

et al., 2017) and theory (e.g. Pavese et al., 1992; Prencipe et al., 2004; Valenzano et al., 2006). As expected, the ATR spectra of the two samples (nanocalcite and IS 120) display three major absorption bands in the investigated frequency range (Fig. 2). They occur at $\sim 712, \sim 873$ and $\sim 1410 \mathrm{~cm}^{-\mathrm{P}}$ and are related to the internal vibrational modes of the carbonate groups, referred to as $v_{4}, v_{2}$ and $v_{3}$, respectively (Hellwege et al., 1970; White, 1974). The IR-active $v_{2}$ mode corresponds to the oscillating motion of the carbon atom perpendicularly to the $\mathrm{CO}_{3}$ plane. It belongs to the $A_{\mathrm{u}}$ representation and its polarization is parallel to the three-fold $c$-axis of the calcite structure (extraordinary ray). A weak and narrow band related to the vibration of the ${ }^{13} \mathrm{CO}_{3}$ isotopic species is also observed at $848 \mathrm{~cm}^{-1}$ (Sterzel \& Chorinsky, 1968; Belousov et al., 1970; White, 1974; Lane, 1999; Floquet et al., 2015; Balan et al., 2017). The IR-active $v_{3}$ and $v_{4}$ modes correspond to anti-symmetric stretching and bending modes of $\mathrm{CO}_{3}$ groups, respectively. They belong to the $E_{\mathrm{u}}$ representation and are polarized perpendicularly to the $c$-axis of calcite (ordinary ray). As previously noticed (e.g. Balan et al., 2017; Griffiths et al., 2018), the intense bands related to the $v_{2}$ and $v_{3} \mathrm{CO}_{3}$ modes are comparatively broader than the other absorption bands. In the Ge IRE spectra, both bands display an asymmetric shape with a steeper decrease in intensity toward lower wavenumbers. Their width coincides with the splitting between transverse optical (TO) and longitudinal optical (LO) frequencies $\left(142 \mathrm{~cm}^{-1}\right.$ and $18 \mathrm{~cm}^{-1}$ for the $v_{3}$ and $v_{2} \mathrm{CO}_{3}$ bands, respectively; White, 1974). In the diamond IRE spectra, larger widths and noticeably different shapes are observed (Fig. 2), an effect particularly obvious for the IS 120 sample. In this specific case, the $v_{3} \mathrm{CO}_{3}$ band displays a tail toward the lower wavenumbers, reducing its asymmetry. Significant 

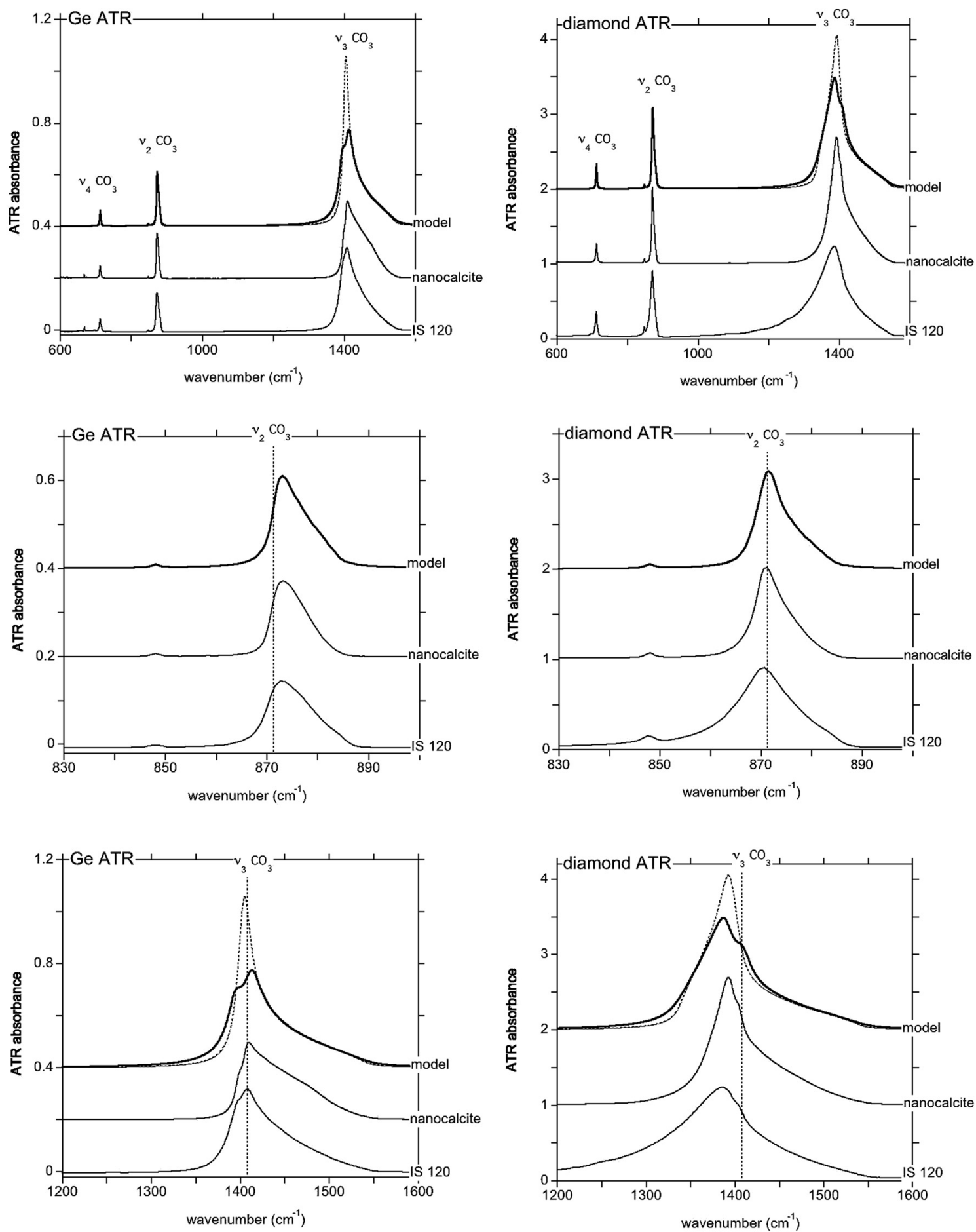

Fig. 2. Experimental and theoretical ATR spectra of calcite. Left and right panels correspond to spectra recorded with a Ge and a diamond IRE, respectively. The top panels display the spectra in the region of interest $\left(600-1600 \mathrm{~cm}^{-1}\right)$ whereas other panels correspond to enlarged views of the $v_{2} \mathrm{CO}_{3}$ (middle) and $v_{3} \mathrm{CO}_{3}$ (bottom) bands. The vertical dotted lines correspond to the TO frequency of the modes. Note the splitting observed near the top of the $v_{3} \mathrm{CO}_{3}$ bands. The model spectrum reported as a dotted curve has been obtained using the classical expression (Eqs. (1) and (2)) of the dielectric properties of calcite whereas that reported as a thicker continuous line has been obtained using a semi-quantum expression (Eq. (3)) for the contribution of the $v_{3} \mathrm{CO}_{3}$ mode. Both models give identical spectra for the $v_{2}$ and $v_{4} \mathrm{CO}_{3}$ bands.

modifications of the relative intensity and shape of this band are also observed as a function of grinding time for times lower than 60 min (Fig. 3).
It is noteworthy that the $v_{3} \mathrm{CO}_{3}$ band of the two investigated samples displays additional features leading to its apparent splitting near its maximum in both the Ge and 

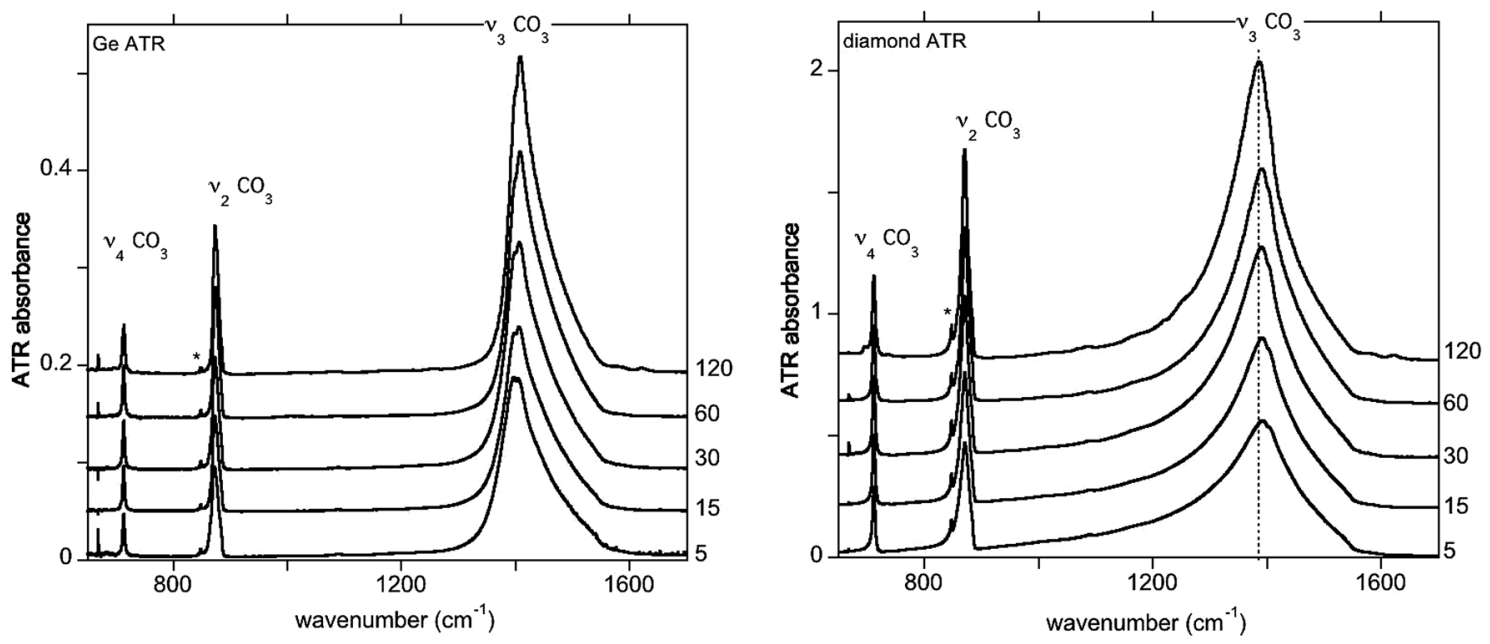

Fig. 3. ATR spectra of the ground Iceland spar sample for grinding times ranging from 5 to 120 min (grinding times are reported on the right of the panels). The weak $v_{2}{ }^{13} \mathrm{CO}_{3}$ band is indicated by a star. Note the relative changes in the intensity and shape of the $v_{3} \mathrm{CO}_{3}$ band as a function of grinding time, which are more prominent in the ATR spectrum recorded with a diamond IRE. In the diamond IRE spectra, the full-width at half maximum (FWHM) of the $v_{3} \mathrm{CO}_{3}$ band decreases from $149 \mathrm{~cm}^{-1}$ to $100 \mathrm{~cm}^{-1}$ from 5 to $120 \mathrm{~min}$ of grinding,

diamond IRE spectra (Fig. 2). In the Ge IRE spectra, the band maximum is located at 1409 and $1408 \mathrm{~cm}^{-1}$ whereas a shoulder is observed at 1400 and $1398 \mathrm{~cm}^{-1}$, for the nanocalcite and IS 120 sample, respectively. On the contrary, in the diamond IRE spectra, the band maximum appears at 1392 and $1386 \mathrm{~cm}^{-1}$ for the nanocalcite and IS 120 sample, respectively, and a shoulder appears on the high-frequency side at $1404 \mathrm{~cm}^{-1}$ for both samples. The shape of the $v_{3} \mathrm{CO}_{3}$ band is thus significantly different depending whether the spectrum is recorded with a $\mathrm{Ge}$ or diamond IRE. The presence of this apparent splitting is at first sight surprising and cannot be explained by a simple oscillator model.

\subsection{Theoretical ATR spectra of calcite}

The modeling strategy adopted in the present study is inspired by that used by Balan et al. (2002) to model specific features in the ATR spectra of chrysotile asbestos and has been exposed in details by Aufort et al. (2016). The theoretical ATR spectrum is obtained by computing the frequency-dependent Fresnel reflection coefficient of an unpolarized infrared beam at a $45^{\circ}$ incidence angle of the interface between the IRE, defined as a homogeneous isotropic medium with constant refractive index $(n=2.4$ for diamond and $n=4$ for $\mathrm{Ge}$ ), and the sample. Moderate polarization effects related to the optical elements of the spectrometer lead to a minor change $(\sim 10 \%)$ of the spectrum intensity not affecting the conclusions of the study. The powder sample is described by an isotropic dielectric function computed for a mixture of spherical calcite particles and an embedding medium corresponding to the empty porosity $(n=1)$. The dielectric function is obtained using the Bruggeman effective medium model (Bruggeman, 1935) which considers that both the particles and the empty domains are inserted in the effective medium, leading to a self-consistent relation between the effective medium properties and those of individual constituents. The powder sample is considered as infinitely thick and homogeneous at the length scale of the experiment, typically requiring particle sizes smaller than a few $\mu \mathrm{m}$. This approach was previously validated for uniaxial materials by modeling the ATR spectra of apatite (Aufort et al., 2016).

The uniaxial dielectric tensor of bulk calcite is obtained using previously proposed expressions relating its dielectric response to its microscopic vibrational and dielectric properties (e.g. De Sousa Meneses et al., 2004; Posch et al., 2007). In an initial modeling attempt, it was built from the microscopic vibrational and dielectric properties of calcite using a usual formalism:

$\varepsilon_{\alpha}(\omega)=\varepsilon_{\alpha}(\infty)+\sum_{j} \varepsilon_{j, \alpha}(\omega)$

where $\alpha$ indicates the ordinary or extraordinary polarization and $\varepsilon_{\alpha}(\infty)$ is a frequency-independent term accounting for the electronic polarizability. The additive contribution of each IR active vibrational mode is described by a Lorentz-type resonant term:

$\varepsilon_{j, \alpha}(\omega)=\frac{A_{j, \alpha}^{2}}{\left(\omega_{j}^{2}-\omega^{2}-i \omega \Gamma_{j}\right)}$

where $A_{j}, \alpha^{2}, \omega_{j}$ and $\Gamma_{j}$ are the mode oscillator strength, the transverse optical (TO) frequency, and damping of mode $j$, respectively. These parameters (Table 1 ) and the electronic terms $\left(\varepsilon_{\text {ordinary }}(\infty)=2.6\right.$ and $\left.\varepsilon_{\text {extraordinary }}(\infty)=2.3\right)$ were obtained from single-crystal reflectivity data by Posch et al. (2007). Some of the TO frequencies and damping parameters $\Gamma_{j}$ (Table 1) were slightly modified to better match experimental observations but are still very close to those of Posch et al. (2007).

The peculiar asymmetry of the ATR bands is closely related to the dielectric properties of the effective medium. As previously observed by Kendrick \& Burnett (2016) and Aufort et al. (2016), these dielectric properties not only 
Table 1. Parameters of the classical model of the calcite dielectric tensor (Eq. (2)). Parameters of Posch et al. (2007) for the $v_{2}, v_{3}$ and $v_{4}$ bands are reported in parenthesis when they differ from the present ones.

\begin{tabular}{lcccc}
\hline Symmetry & Mode & $\omega_{j}\left(\mathrm{~cm}^{-1}\right)$ & $A_{j, \mathrm{a}}($ arb. units $)$ & $\Gamma_{j}\left(\mathrm{~cm}^{-1}\right)$ \\
\hline$A_{\mathrm{u}}$ & $v_{2}$ & $871.2(872.0)$ & 250.8 & $3.0(2.09)$ \\
& $v_{2}{ }^{*}$ & 848.0 & 30.0 & 2.1 \\
$E_{\mathrm{u}}$ & $v_{3}$ & 1407.4 & 1039.6 & $15.0(14.8)$ \\
& $v_{4}$ & $712.6(712.0)$ & 71.4 & $3.0(4.0)$ \\
\hline
\end{tabular}

${ }^{* 13} \mathrm{CO}_{3}$ isotopic species.

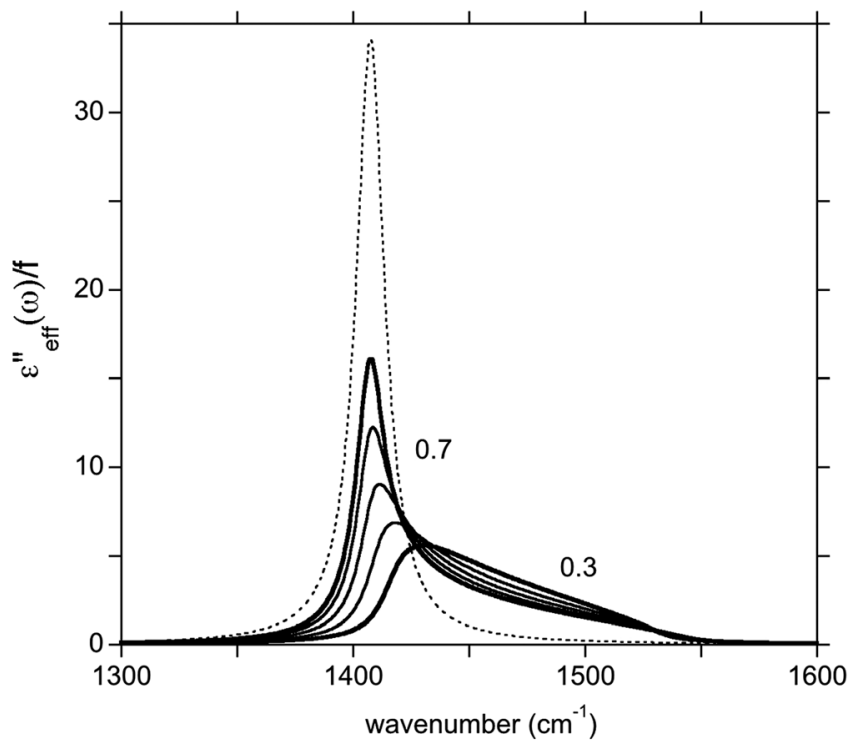

Fig. 4. Imaginary part of effective dielectric function in the $v_{3} \mathrm{CO}_{3}$ range computed using the classical expression of the calcite dielectric tensor and the Bruggeman model for a volume fraction of calcite varying from 0.3 to 0.7 by steps of 0.1 . The effective dielectric functions have been divided by the corresponding volume fraction. The imaginary part of the dielectric function of pure calcite corresponding to the $v_{3} \mathrm{CO}_{3}$ mode is reported for comparison (dotted line).

depend on those of the bulk material but are also strongly dependent on the relative volume fraction occupied by the solid and the empty porosity (Fig. 4). For vanishingly low solid fractions, the dielectric function related to a strongly absorbing mode displays a single symmetric resonance at a frequency coinciding with that of the isolated sphere. For intermediate solid fractions, the imaginary (absorptive) part of the dielectric function extends from the transverse optical (TO) to the longitudinal optical (LO) frequencies of the mode. Its asymmetry increases with increasing solid fractions, converging toward a sharp peak at the TO frequency for the larger fractions of solid.

Assuming a spherical shape of particles, the solid fraction is the only free parameter of the model. Considering that the shape of absorption bands in the ATR spectra recorded with a Ge IRE are similar to that of the resonances in the imaginary part of the dielectric function (Aufort et al., 2016), a volume fraction of solids of $f=0.55$ provides the best agreement between theory and experiment (Fig. 2). With this set of microscopic (Table 1) and macroscopic parameters, the model spectra account for the relative intensity of the bands and variations of the overall intensity observed between spectra recorded with a Ge and with a diamond IRE. The model also reproduces the asymmetric shape of the $v_{2} \mathrm{CO}_{3}$ band (Fig. 2) and the moderate down-shift of its maximum in the diamond IRE spectrum.

Compared with the nanocalcite sample, a poorer agreement is observed on the low-frequency side of the $v_{2} \mathrm{CO}_{3}$ and $v_{3}$ $\mathrm{CO}_{3}$ bands in the diamond ATR spectrum of the IS 120 sample. Both bands display a low-frequency tail that extends to significantly lower frequencies than those of the model spectra. This specific difference is most likely related to grain-size inhomogeneities in this mechanically ground sample. As exposed in details by Belali et al. (1995), anomalous dispersion may cause the experiment to move from the ATR regime to the specular reflection regime for strongly absorbing materials. In this regime, only a fraction of the IR light is reflected at the interface between the sample and the IRE, whereas the other fraction is transmitted and propagates in the powder sample. For an IRE of indice $n$ and an incidence angle of $45^{\circ}$, total reflection of the beam on a flat interface with a medium of index $n_{\mathrm{s}}$ occurs when $n_{\mathrm{s}}<\sin \left(45^{\circ}\right) n$. Accordingly, the specular reflection domain is increased in spectra recorded using an IRE with a lower refractive index, such as diamond $(n=2.4) v s$. Ge $(n=4)$ (Belali et al., 1995). In an inhomogeneous sample containing particles with a size larger than the characteristic length scale of mid-IR $\left(7.1 \mu \mathrm{m}\right.$ at $\left.1400 \mathrm{~cm}^{-1}\right)$, local dielectric properties can be closer to those of the pure material than to the average ones (Fig. 4), enhancing anomalous dispersion effects with respect to those modeled for the homogeneous effective medium. For pure calcite, the specular reflection domain associated to the $v_{3} \mathrm{CO}_{3}$ band extends up to $\sim 700 \mathrm{~cm}^{-1}$ and $\sim 60 \mathrm{~cm}^{-1}\left(45 \mathrm{~cm}^{-1}\right.$ and $5 \mathrm{~cm}^{-1}$ for the $v_{2} \mathrm{CO}_{3}$ band) below the transverse optical frequency for a diamond and Ge IRE, respectively. These values are well consistent with the extension of the low-frequency side of the $\mathrm{v}_{2} \mathrm{CO}_{3}$ and $v_{3} \mathrm{CO}_{3}$ bands observed in the ATR spectra of the IS sample ground for 5 min (Fig. 3; see also Kristova et al., 2015) and this effect explains their narrowing as a function of grain size (Udvardi et al., 2017) or grinding time (Fig. 3), especially in the spectra recorded with a diamond IRE.

Concerning the $v_{3} \mathrm{CO}_{3}$ band, a peculiar disagreement between theory and experiment is observed close to its maximum (Fig. 2). Although the theoretical band still extends between the TO and LO frequencies, the model overestimates the band intensity close to the TO mode frequency in the Ge IRE spectrum and fails to reproduce the splitting observed in the same region in both Ge and diamond IRE spectra (Fig. 2). Various physical effects can explain the occurrence of additional structures in the shape of strong infrared absorption bands (e.g. Ruppin \& Englman, 1970; Poduska et al., 2011). For example, depolarization fields occurring in small particles shift their resonant frequencies toward values higher than the TO frequency, leading to so-called surface modes. Bulk phonon polaritons related to the coupling between vibrational excitations and light 
propagation in crystals may lead to additional features observed at frequencies lower than the TO one and enhanced for larger particle sizes. In the present case, the anomalous features are observed at frequencies lower than the TO frequency and occur in both samples. As the nanocalcite sample is made of sub-micrometer sized particles, this rules out a potential origin related to the two above mentioned effects.

It is noteworthy that the calcite reflectivity spectrum reported by Posch et al. (2007) also displays a structure close to the top of the $v_{3} \mathrm{CO}_{3}$ band. These reflectivity measurements were performed on calcite crystals with a peculiar shape differing from the usual rhombohedral shape and exhibiting growth faces parallel to the symmetry axis (Posch et al., 2007). The observed structure is not accounted for by the classical model (Eqs. (1) and (2)) used to describe the dielectric properties of bulk calcite. Similar structures, not predicted by a group-theory analysis of the crystal properties, are observed in the reflectance spectrum of strongly absorbing materials with a large splitting between LO and TO frequencies, such as MgO (De Sousa Meneses et al., 2004). As shown by De Sousa Meneses et al. (2004), these features can be attributed to a frequency dependence of the phonon damping in the expression of the complex dielectric function. According to this semi-quantum approach, the contribution of the IR active optical vibration $j$ to the dielectric response of the material becomes (Cowley, 1963):

$\varepsilon_{j, \alpha}(\omega)=\frac{A_{j, \alpha}^{2}}{\omega_{j}^{2}-\omega^{2}-i \omega_{j} \Gamma_{j}(\omega)}$

where a frequency dependence of the damping parameter $\Gamma_{j}(\omega)$ is allowed. Whereas the $\Gamma_{j}$ are approximated as frequency-independent constants in the standard expression (Eq. (2)), the frequency dependence of $\Gamma_{j}(\omega)$ plays a relevant role in the present situation. It is worth to add that the $\Gamma_{j}(\omega)$ function is closely related to the phonon selfenergy and should meet the causality criteria, which implies that it displays a real and an imaginary part which are related to each other through Kramers-Kronig transforms (Rammer, 2007).

Following De Sousa Meneses et al. (2004), the most important characteristics of $\Gamma_{\mathrm{j}}(\omega)$ can be described as a sum of a small number of more or less symmetric peaks. In the present study, we consider a model damping function $\Gamma(\omega)$ to describe the behavior of the $v_{3} \mathrm{CO}_{3}$ band. Its real part is built as the sum of a frequency-independent damping term $\left(\Gamma_{0}\right)$ and a Gaussian peak centered at frequency $\omega_{\mathrm{SE}}$ :

$\operatorname{Re}(\Gamma(\omega))=\Gamma_{0}+2 A \exp \left(-\left(\frac{\omega-\omega_{\mathrm{SE}}}{\gamma}\right)^{2}\right)$

where $A$ and $\gamma$ are the amplitude and half-width at halfmaximum of the Gaussian peak, respectively (Fig. 5). In the actual implementation, this expression was slightly modified to meet the causality criteria (Keefe, 2001) and its related imaginary part $(\operatorname{Im}(\Gamma(\omega))$ was obtained by a Kramers-Kronig transform of the real part, as in Eqs. (18)-(20) of De Sousa Meneses et al. (2004).

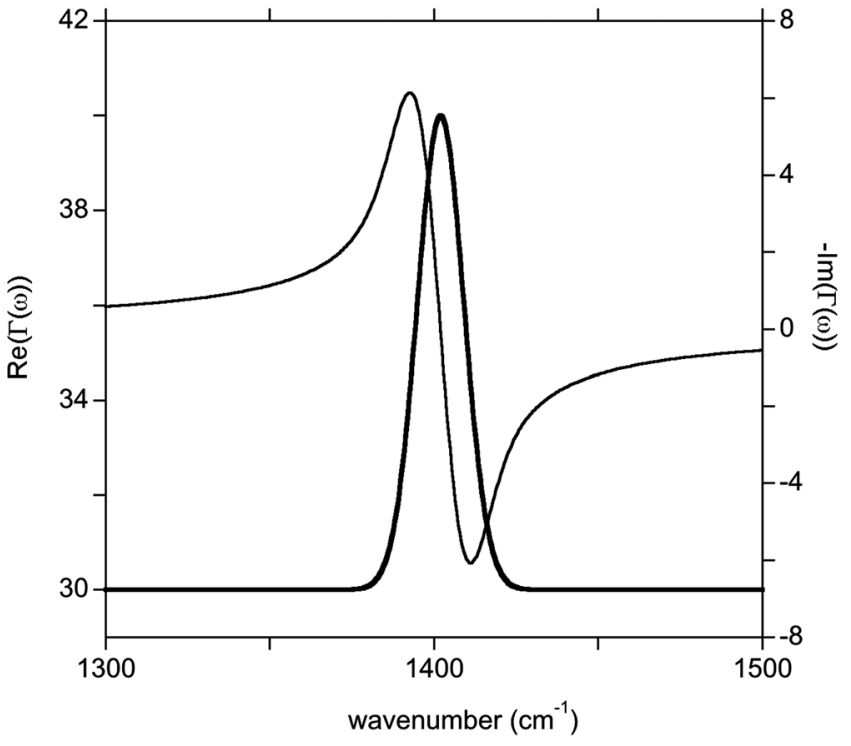

Fig. 5. Real (thick line) and imaginary (thin line) part of the model frequency-dependent function describing the damping of the $v_{3} \mathrm{CO}_{3}$ vibrational excitation in calcite (Eq. (4)).

The parameters describing the $\Gamma(\omega)$ function (Eq. (4)) were set to $\omega_{\mathrm{SE}}=1402 \mathrm{~cm}^{-1}, A=5 \mathrm{~cm}^{-1}, \gamma=10 \mathrm{~cm}^{-1}$ and the constant term to $\Gamma_{0}=30 \mathrm{~cm}^{-1}$ to reproduce the salient features of the ATR spectra. Compared with the classical expression of the dielectric tensor, the modified dielectric tensor accounts for the main features of the $v_{3} \mathrm{CO}_{3}$ band observed in the ATR spectra (Fig. 2). More specifically, it reproduces the splitting observed close to the band maximum, the significant intensity reduction at the TO frequency observed in the Ge IRE spectrum and the change in band shape with a shoulder observed on the high- or low-frequency side of the maximum in the spectra recorded with the diamond or Ge IRE, respectively.

It is noteworthy that the $v_{3} \mathrm{CO}_{3}$ band in the reflectivity spectrum computed with the modified model (Fig. 6) also displays a feature consistent with the experimental observations of Posch et al. (2007). Compared with the data of Posch et al. (2007), the present simulation displays however a decrease in the overall reflectivity in the reststrahlen range of the band due to a constant damping $\left(\Gamma_{0}=30 \mathrm{~cm}^{-1}\right)$ larger than that used in the classical model. This larger value was introduced to improve the agreement with the present ATR spectra. It does not necessarily correspond to a change in the homogeneous broadening mechanism of the band in the different samples but more likely reflects imperfections of the simple effective medium model and frequency-dependent damping function to provide a full account of the properties of real samples.

\section{Discussion}

The present results point out peculiar features of the IRactive $v_{3} \mathrm{CO}_{3}$ mode in calcite related to a resonance occurring at $\sim 1402 \mathrm{~cm}^{-1}$ in its damping function. Even 


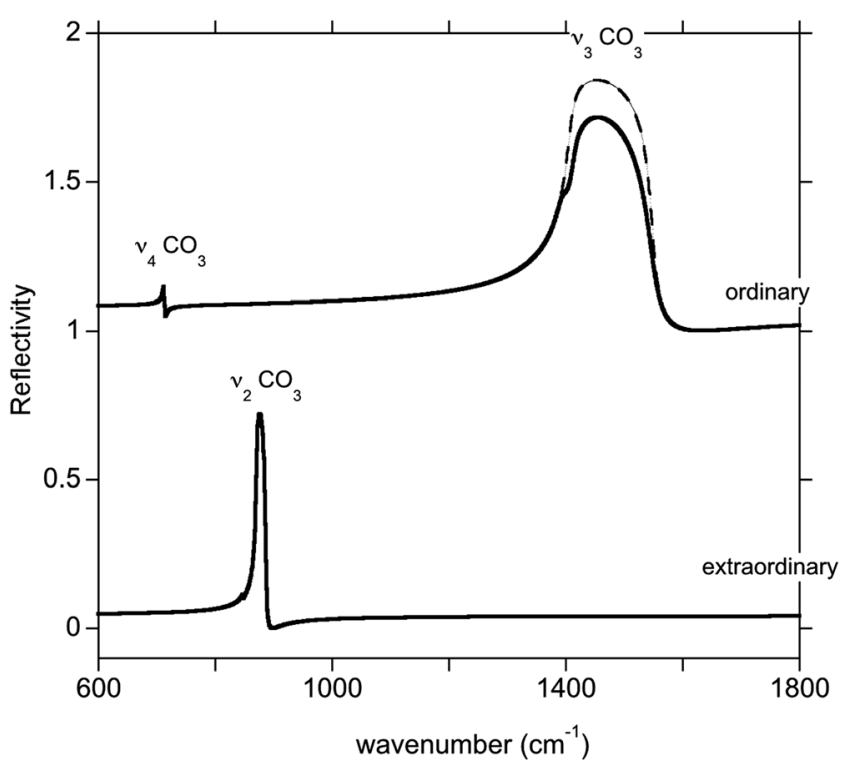

Fig. 6. Theoretical reflectivity of calcite. The reflectivity reported as a dotted curve was computed using the classical model with the parameters reported in Table 1 . The continuous curve was obtained using a semi-quantum expression for the contribution of the $v_{3} \mathrm{CO}_{3}$ mode. The ordinary spectra were vertically shifted by one unit.

though these features can be inferred from previously reported reflectance data (Posch et al., 2007), to our knowledge they remained unnoticed in most previous infrared spectroscopic studies of calcite. They distinctly appeared in the present study due to the crystalline quality of the investigated samples and to the specific shape of absorption bands in ATR spectra, enhancing the spectrum intensity in regions close to the TO frequencies of strongly absorbing modes. It is worth to note that they were not observed in the ATR spectra of the biogenic calcite sample investigated by Balan et al. (2017). In this case, they are masked by the inhomogeneous broadening of infrared lines due to the occurrence of significant concentrations of $\mathrm{Mg}, \mathrm{Na}, \mathrm{Sr}$ and sulfate chemical impurities in this sample.

These features are related to the frequency dependence of the damping function. To understand this point, we remind that the intrinsic component of phonon damping is due to phonon-phonon scattering and its expression can be interpreted as resulting from the Fermi Golden rule of quantum mechanics. Let us consider an IR-active phonon labeled by a branch index $j$ : the wavevector of the phonon is $\mathbf{0}$ and, because of momentum conservation, it can decay in two phonons with equal and opposite momentum q. At zero temperature, the real part of the damping related to this three-phonon process is proportional to (Menéndez \& Cardona, 1984):

$\operatorname{Re}\left(\Gamma_{j}(\omega)\right)=\sum_{\mathbf{q}, k, l}\left|V_{\mathbf{q} k l}\right|^{2} \delta\left(\omega-\omega_{\mathbf{q} k}-\omega_{-\mathbf{q} l}\right)$

where the sum is performed on all the phonon momenta $\mathbf{q}$ in the Brillouin zone and for all the phonon branches $k$ and $l . V_{\mathbf{q} k l}$ is the scattering coefficient. The Dirac delta distribution in the right term implements the conservation of energy in the process. A more general formulation should include coalescence processes, temperature dependence of the phonon occupation and, possibly, higher-order scattering processes, without however altering the following considerations. It is important to notice that the conservation of energy in Eq. (5) is applied to $\omega$, which is the frequency of the light probe, and not to $\omega_{j}$, which is the intrinsic TO frequency of the phonon. This is because, in the IR experiment, the atoms are actually vibrating at the frequency $\omega$ imposed by the electric field associated with the probing light. In the cases where $\Gamma_{j}(\omega)$ presents a non-trivial smooth behavior, this dependence can lead to measurable effects (usually called multi-phonon effects). In particular, it is common to observe in many materials intense peaks in the phonon density of states corresponding to phonons with a small dispersion in a certain volume of the Brillouin zone. Because of the energy conservation, a decay process in which the final states $(\mathbf{q} k$ and $-\mathbf{q} l$ in Eq. (5)) belong to such a phonon branch will be activated only for certain specific values of the frequency $\omega$ of the initial state. The resulting $\Gamma_{j}(\omega)$ could possibly present sharp peaks at those $\omega$ values.

A potential origin of a peak at $1402 \mathrm{~cm}^{-1}$ in the damping function of the $v_{3} \mathrm{CO}_{3}$ excitation can be proposed by examining its coupling to the phonon density of states of calcite. According to the group-theory analysis of Delfyett et al. (1989), five possible three-phonon and eighteen four-phonon decay paths are allowed by symmetry for a mode with $E_{\mathrm{u}}$ symmetry. Among these paths, only those ensuring energy and momentum conservation actually exist. Limiting the discussion to three-phonon processes, only the decay of the $E_{\mathrm{u}}$ excitation into the non-dispersive totally symmetric stretching mode at $1086 \mathrm{~cm}^{-1}$ ( $A_{1 \mathrm{~g}}$ symmetry) and a LO external mode with $E_{\mathrm{u}}$ symmetry and a dispersion ranging between 381 and $320 \mathrm{~cm}^{-1}$ (White, 1974; Delfyett et al., 1989) could meet these criteria.

Finally, it is worth to note that the origin of the broadening of the strongly absorbing bands differ from that discussed by Griffiths et al. (2018). As pointed out by Griffiths et al. (2018), transmission spectra of strongly absorbing materials recorded after dilution in an IR transparent medium are broadened due to a combination of saturation effects and inhomogeneities of the preparation resulting in voids in the analyzed sample. In ATR spectra recorded with a highly refractive IRE, such as Ge, the primary cause of broadening is instead related to the electrostatic interactions within and between particles. These interactions are important for intermediate volume fractions of solids, such as those observed in standard powder samples, and are quantitatively accounted for by the Bruggeman effective medium model (Aufort et al., 2016). Additional causes of broadening related to anomalous dispersion are observed in ATR spectra recorded with a less refractive IRE medium such as diamond. They become particularly significant in samples displaying grain sizes larger than the characteristic length scale of mid-IR radiation, typically a few micrometers. The IRE choice for the ATR-FTIR spectroscopic analysis of minerals thus implies a trade-off between an improved signal/noise 
ratio but a greater sensitivity to broadening sources of diamond $v s$. more refractive materials such as Ge.

\section{Conclusion}

The present results underline the benefit of coupling experimental and modeling approaches in the infrared spectroscopic study of minerals. A single and simple frequency-dependent damping function for the $v_{3} \mathrm{CO}_{3}$ mode in calcite can account for spectroscopic observations performed using three different experimental settings in the infrared range (ATR of powders with Ge and diamond IRE, specular reflectance of a single crystal), each setting leading to a specific spectrum. This combination of approaches makes it possible to discriminate among physically different processes affecting the shape of infrared absorption bands, some being related to the longrange nature of electrostatic interactions in polar materials and others being related to atomic-scale anharmonic interactions between vibrational modes. The approach followed in the present study is generally applicable and could be extended to a large number of powder minerals and materials.

Acknowledgements: We thank Camille Bourgeoisat (UFR TEB, Sorbonne Université) for her contribution to this work. We thank I. Estève and the IMPMC microscopy platform for the SEM images. Support by M. Guillaumet, K. Béneut and the IMPMC spectroscopy platform is acknowledged. We thank two anonymous reviewers for their pertinent comments. This work has been supported by the French National Research Agency through the CARBORIC (ANR-13-BS06-0013-06) project and by French state funds within the framework of the Cluster of Excellence MATISSE led by Sorbonne Universités.

\section{References}

Amma, S., Luo, J., Pantano, C.G., Kim, S.H. (2015): Specular reflectance (SR) and attenuated total reflectance (ATR) infrared (IR) spectroscopy of transparent flat glass surfaces: A case study for soda lime float glass. J. Non-Cryst. Sol., 428, 189-196.

Aufort, J., Ségalen, L., Gervais, C., Brouder, C., Balan, E. (2016): Modeling the attenuated total reflectance infrared (ATR-FTIR) spectrum of apatite. Phys. Chem. Minerals, 43, 615-626.

Aufort, J., Lebon, M., Gallet, X., Ségalen, L., Gervais, C., Brouder, C., Balan, E. (2018): Macroscopic electrostatic effects in the ATR-FTIR spectra of modern and archaeological bones. Am. Mineral., 103, 326-329.

Balan, E., Mauri, F., Lemaire, C., Brouder, C., Guyot, F., Saitta, A.M., Devouard, B. (2002): Multiple ionic plasmon resonances in naturally-occurring multiwall nanotubes: Infrared spectra of chrysotile asbestos. Phys. Rev. Lett., 89, 177401.

Balan, E., Aufort, J., Pouillé, S., Dabos, M., Blanchard, M., Lazzeri, M., Rollion-Bard, C., Blamart, D. (2017): Infrared spectroscopic study of sulfate-bearing calcite from deep-sea bamboo coral. Eur. J. Mineral., 29, 397-408.

Belali, R., Vigoureux, J.-M., Morvan, J. (1995): Dispersion effects on infrared spectra in attenuated total reflection. J. Opt. Soc. Amer. B, 12, 2377-2381.

Belousov, M.V., Pogarev, D.E., Shultin, A.A. (1970): Band intensity of extraplanar $v_{2}$ vibration of $\mathrm{CO}_{3}^{2-}$ and $\mathrm{NO}_{3}^{-}$ions in crystals having calcite and aragonite structure. Sov. Phys. Sol. State, 11, 2185-2186.

Boulet-Audet, M., Buffeteau, T., Boudreault, S., Daugey, N., Pézolet, M. (2010): Quantitative determination of band distortions in diamond attenuated total reflectance infrared spectra. $J$. Phys. Chem. B, 114, 8255-8261.

Bruggeman, D.A.G. (1935): Berechnung verschiedener physikalischer Konstanten von heterogenen Substanzen. I. Dielektrizitätskonstanten und Leitfähigkeiten der Mischkörper aus isotropen Substanzen. Ann. Phys., 416, 665-679.

Cowley, R.A. (1963): The lattice dynamics of an anharmonic crystal. Adv. Phys., 12, 421-480.

Delfyett, P.J., Dorsinville, R., Alfano, R.R. (1989): Multiphonon dephasing of the $1086-\mathrm{cm}^{-1}$ mode in calcite. Phys. Rev. B, 39, 3845-3853.

De Sousa Meneses, D., Brun, J.-F., Echegut, P., Simon, P. (2004): Contribution of semi-quantum dielectric function models to the analysis of infrared spectra. Appl. Spectrosc., 58, 969-974.

Dignam, M.J. \& Mamiche-Afara, S. (1988): Determination of the spectra of the optical constants of bulk phases via Fourier transform ATR. Spectrochim. Acta, 44A, 1435-1442.

Farmer, V.C. (1974): The infrared spectra of minerals, Mineralogical Society, London.

Floquet, N., Vielzeuf, D., Ferry, D., Ricolleau, A., Heresanu, V., Perrin, J., Laporte, D., Fitch, A.N. (2015): Thermally induced modifications and phase transformations of red coral Mg-calcite skeletons from infrared spectroscopy and high resolution synchrotron powder diffraction analyses. Cryst. Growth Des., 15, 3690-3706.

Gueta, R., Natan, A., Addadi, L., Weiner, S., Refson, K., Kronik, L. (2007): Local atomic order and infrared spectra of biogenic calcite. Angew. Chem. Int. Ed., 46, 291-294.

Griffiths, P.R., Fries, B.E., Weakley, A.T. (2018): On the widths of the bands in the infrared spectra of oxyanions. Appl. Spectrosc., 72, 863-869.

Hellwege, K.H., Lesch, W., Plihal, M., Schaack, G. (1970): Zwei-Phononen-Absorptionsspektren und Dispersion der Schwingungszweige in Kristallen der Kalkspatstruktur. Zeit. Phys., 232, 61-86.

Huang, J.B. \& Urban, M.W. (1992): Evaluation and analysis of attenuated total reflectance FT-IR spectra using Kramers-Kronig transforms. Appl. Spectrosc., 46, 1666-1672.

Jenkins, D.M., Holmes, Z.F., Ishida, K., Manuel, P.D. (2018): Autocorrelation analysis of the infrared spectra of synthetic and biogenic carbonates along the calcite-dolomite join. Phys. Chem. Minerals, 45, 563-574.

Kendrick, J. \& Burnett, A.D. (2016): PDielec: The calculation of infrared and terahertz absorption for powdered crystals. $J$. Comput. Chem., 37, 1491-1504.

Keefe, C.D. (2001): Curvefitting imaginary components of optical properties: Restrictions on the lineshape due to causality. J. Mol. Spectros., 205, 261-268.

Kristova, P., Hopkinson, L.J., Rutt, K.J. (2015): The effect of the particle size on the fundamental vibrations of the $\left[\mathrm{CO}_{3}{ }^{2-}\right]$ anion in calcite. J. Phys. Chem. A, 119, 4891-4897.

Lane, M.D. (1999): Mid infrared optical constants of calcite and their relationship to particle size effects in thermal emission spectra of granular calcite. J. Geophys. Res., 104, 14099-14108.

Lebon, M., Reiche, I., Gallet, X., Bellot-Gurlet, L., Zazzo, A. (2016): Rapid quantification of bone collagen content by ATR-FTIR spectroscopy. Radiocarbon, 58, 131-145.

Loftus, E., Rogers, K., Lee-Thorp, J. (2015): A simple method to establish calcite:aragonite ratios in archaeological mollusc shells. J. Quaternary Sci., 30, 731-735.

McMillan, P.F. \& Hofmeister, A.M. (1988): Infrared and Raman spectroscopy. in "Spectroscopic Methods in Mineralogy and 
Geology. Reviews in Mineralogy", Vol. 18, Mineralogical Society of America, Washington, DC, 513-571.

Menéndez, J. \& Cardona, M. (1984): Temperature dependence of the first-order Raman scattering by phonons in $\mathrm{Si}, \mathrm{Ge}$, and $\alpha-\mathrm{Sn}$ : Anharmonic effects. Phys. Rev. B, 29, 2051-2059.

Pavese, A., Catti, M., Price, G.D., Jackson, R.A. (1992): Interatomic potentials for $\mathrm{CaCO}_{3}$ polymorphs (calcite and aragonite), fitted to elastic and vibrational data. Phys. Chem. Minerals, 19, 80-87.

Poduska, K.M., Regev, L., Boaretto, E., Addadi, L., Weiner, S., Kronik, L., Curtarolo, S. (2011): Decoupling local disorder and optical effects in infrared spectra: Differentiating between calcites with different origins. Adv. Mater., 23, 550-554.

Pokrovsky, O., Pokrovski, G., Schott, J. (2004): Gallium (III) adsorption on carbonates and oxides: X-ray absorption fine structure spectroscopy study and surface complexation modeling. J. Coll. Inter. Sci., 279, 314-325.

Posch, Th., Baier, A., Mutschke, H., Henning, Th. (2007): Carbonates in space: The challenge of low-temperature data. Astrophys. J., 668, 993-1000.

Prencipe, M., Pascale, F., Zicovich-Wilson, C.M., Saunders, V.R., Orlando, R., Dovesi, R. (2004): The vibrational spectrum of calcite $\left(\mathrm{CaCO}_{3}\right)$ : An ab initio quantum-mechanical calculation. Phys. Chem. Minerals, 31, 559-564.

Rammer, J. (2007): Quantum field theory of non-equilibrium states. Cambridge University Press, 552 p.

Ruppin, R. \& Englman, R. (1970): Optical phonons in small crystals. Rep. Prog. Phys., 33, 149-196.
Saldi, G.D., Noireaux, J., Louvat, P., Faure, L., Balan, E., Schott, J., Gaillardet, J. (2018): Boron isotopic fractionation during adsorption by calcite - Implication for the seawater $\mathrm{pH}$ proxy. Geochim. Cosmochim. Acta, 240, 255-273.

Sterzel, W. \& Chorinsky, E. (1968): Die Wirkung schwerer Kohlenstoffisotope auf das Infrarotspektrum von Carbonaten. Spectrochim. Acta, 24A, 353-360.

Udvardi, B., Kovács, I., Fancsik, T., Kónya, P., Bátori, M., Stercel, F., Falus, G., Szalai, Z. (2017): Effects of particle size on the attenuated total reflection spectrum of minerals. Appl. Spectrosc., 71, 1157-1168.

Valenzano, L., Torres, F. J., Doll, K., Pascale, F., Zicovich-Wilson, C.M., Dovesi, R. (2006): Ab initio study of the vibrational spectrum and related properties of crystalline compounds: The case of $\mathrm{CaCO}_{3}$ calcite. Zeit. Phys. Chem., 220, 893-912.

Weiner, S. \& Dove, P.M. (2003): An overview of biomineralization processes and the problem of the vital effect. Rev. Mineral. Geochem., 54, 1-29.

White, W.B. (1974): The carbonate minerals. in "The Infrared Spectra of Minerals", Farmer, V.C., ed. Mineralogical Society, London, 227-284.

Received 25 May 2018

Modified version received 3 July 2018

Accepted 3 July 2018 